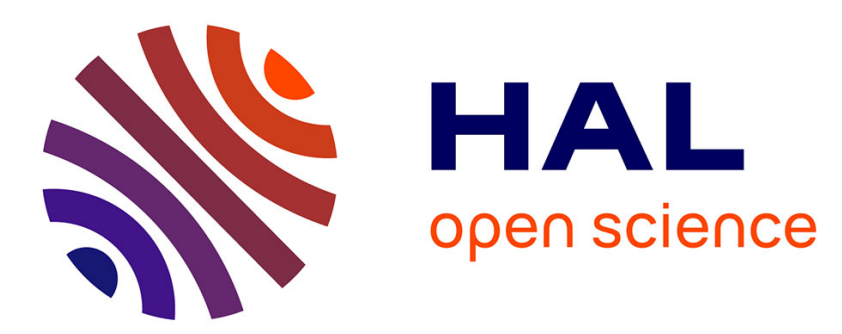

\title{
Gouvernance des sports professionnels: une approche en termes de mutualisation
}

François Fulconis, Nollet Jean, Paché Gilles

\section{To cite this version:}

François Fulconis, Nollet Jean, Paché Gilles. Gouvernance des sports professionnels: une approche en termes de mutualisation. Logistique \& Management, 2016, 24 (3/4), pp.215-225. 10.1080/12507970.2016.1268075 . hal-01449099

\section{HAL Id: hal-01449099 \\ https://hal-amu.archives-ouvertes.fr/hal-01449099}

Submitted on 23 Sep 2019

HAL is a multi-disciplinary open access archive for the deposit and dissemination of scientific research documents, whether they are published or not. The documents may come from teaching and research institutions in France or abroad, or from public or private research centers.
L'archive ouverte pluridisciplinaire HAL, est destinée au dépôt et à la diffusion de documents scientifiques de niveau recherche, publiés ou non, émanant des établissements d'enseignement et de recherche français ou étrangers, des laboratoires publics ou privés. 


\title{
Gouvernance des sports professionnels : une approche en termes de mutualisation
}

\author{
François Fulconis, Jean Nollet \& Gilles Paché
}

To cite this article: François Fulconis, Jean Nollet \& Gilles Paché (2016) Gouvernance des sports professionnels : une approche en termes de mutualisation, Logistique \& Management, 24:3-4, 215-225, DOI: 10.1080/12507970.2016.1268075

To link to this article: https://doi.org/10.1080/12507970.2016.1268075

册 Published online: 09 Jan 2017.

Submit your article to this journal

山 Article views: 37

View Crossmark data \lceil 


\title{
Gouvernance des sports professionnels : une approche en termes de mutualisation
}

\author{
François Fulconis ${ }^{\mathrm{a}}$, Jean Nollet ${ }^{\mathrm{b}}$ et Gilles Pachéc \\ aCentre de Recherche sur le Transport et la Logistique (CRET-LOG), Université d'Avignon et des Pays de Vaucluse, Avignon, France ; \\ bProfessorship Association de la Gestion de la Chaîne d'Approvisionnement (AGCA), HEC Montréal, Montréal, Canada ; 'Centre de \\ Recherche sur le Transport et la Logistique (CRET-LOG), Aix-Marseille Université, Aix-en-Provence, France
}

\begin{abstract}
RÉSUMÉ
Les achats font l'objet d'une abondante littérature depuis plus de trois décennies, ce qui reflète pour cette fonction le glissement d'une vision opérationnelle à une vision stratégique. Ceci se traduit notamment par l'émergence d'une logique "gagnant-gagnant »; par exemple, tout en maintenant la compétition entre elles, des entreprises mettent en œuvre des stratégies de coopétition et mutualisent leurs achats, entre autres pour réduire les coûts logistiques et les délais de mise à disposition des produits, mais aussi pour accroître la qualité de service rendue aux clients. Fort des réflexions menées sur ces phénomènes, l'article aborde de façon originale le domaine sportif sous l'angle des achats. L'objectif est d'examiner comment la notion de mutualisation peut être utile pour mieux comprendre les avantages découlant de l'approche collective des ligues nord-américaines en matière de politique d'achat de joueurs et, plus largement, de gestion du capital humain.
\end{abstract}

\section{Governance of professional sports: using a pooling approach}

\section{ABSTRACT}

For more than three decades, purchasing has been a major area for publication, reflecting the change of perception and in reality from an operational view of purchasing to a strategic one. This has resulted in particular in the emergence of a win-win approach; as an example, while still competing one with another, organisations can also use coopetition and pooling strategies with objectives such as logistical cost and delivery time reduction, but also to deliver quality improvements to the customer. Using a similar line of thought, this article deals in an original way with the world of sports through a purchasing lens by examining how the pooling concept could be useful to understand better the advantages stemming from the North American leagues' strategy of player contract purchases, and more globally, of human capital management.

\section{MOTS CLÉS}

Capital humain ; coopétition ; mutualisation sport professionnel ; stratégies collectives ; stratégies d'achat

\section{KEYWORDS}

Human capital; coopetition; pooling; professional sport; collective strategies; purchasing strategy

\section{Introduction}

Les stratégies d'achat sont habituellement abordées dans la perspective des produits ou des services, tout particulièrement comme levier afin de conforter un avantage concurrentiel pour la seule organisation acheteuse. En revanche, les stratégies d'acquisition de joueurs par les clubs professionnels n'ont guère donné lieu à des réflexions dans le champ du management, et encore moins des achats. Pourtant, dans l'analyse économique des sports professionnels, les joueurs les plus talentueux sont une ressource rare et difficilement imitable, qui est au cœur des performances sportives mais aussi financières des clubs (par exemple, à travers la vente des droits TV et des produits dérivés). En examinant notamment le football professionnel, qui fait désormais l'objet de recherches scientifiques (Zoudji et Rey 2015), il est possible de constater sur le plan européen que le marché primaire des stars donne lieu à une surenchère continue de la part de quelques clubs, systématiquement les plus riches du Continent. Doit-on rappeler ici le salaire astronomique négocié en janvier 2016 par Lionel Messi (Barcelone), à savoir 40 millions d'euros versés chaque année par son club, pour les quatre prochaines saisons ? Les stratégies d'achat de joueurs sont ainsi cohérentes avec le modèle du capital humain, et la captation de ressources rares et difficilement imitables est une nécessité pour que le business model d'un club puisse réussir.

En revanche, un tout autre modèle constitue la base des ligues nord-américaines, et mérite une étude approfondie compte tenu de leurs succès récurrents. Le modèle nord-américain des ligues fermées (dénommées « franchises ») est fondé sur des règles collectivisées dans la mise en œuvre de stratégies d'achat. Neale (1964) souligne d'ailleurs très tôt combien la coopération entre clubs

CONTACT Gilles Paché gilles.pache@univ-amu.fr

(c) 2017 KEDGE Business School 
(franchises) est essentielle au fonctionnement efficace d'une ligue fermée. Pour simplifier, par la règle de la draft, ce sont les clubs les plus mal placés à la fin de la saison $\mathrm{n}$ qui sont prioritaires pour le recrutement des nouveaux talents de la saison $n+1$. L'objectif est d'éviter que la ligue fermée soit condamnée à voir une poignée de clubs remporter un titre année après année. Avec la règle de la draft, un club réputé « faible » la saison n peut se voir offrir l'opportunité de briller à la saison $n+1$, créant alors un phénomène de surprise très positif pour les audiences; ces dernières génèrent des droits TV que tous les clubs de la ligue se répartissent de manière équilibrée. En d'autres termes, la stratégie collectivisée d'achat de joueurs dans les ligues nord-américaines participe à un autre modèle : c'est la ligue elle-même qui cherche à faire fructifier ses revenus, et moins chacun de ses clubs franchisés.

L'article souhaite ainsi aborder un secteur peu étudié en management d'entreprise, et lorsqu'il est étudié, uniquement sous des dimensions marketing et financières. L'angle d'attaque est ici celui du management des achats (de joueurs) et des stratégies de mutualisation de ressources pour améliorer collectivement la position d'une industrie, ce qui renvoie au paradigme de l'action concurrentielle concertée (Le Roy et Yami 2009). Il est ainsi possible de montrer que des grilles de lecture robustes peuvent s'appliquer à cette réalité singulière du management des organisations. Dès lors que l'on considère, à la suite de van Weele (2014), que les achats ont pour vocation, dans toute organisation publique ou privée, d'acquérir les produits ou services nécessaires à son activité commerciale dans les meilleures conditions de compétitivité, la question de la gestion des achats de joueurs par des clubs professionnels ayant un objectif affiché de rentabilité se pose avec acuité. Au demeurant, Lonsdale (2004) n'hésite pas à examiner le sport professionnel, et singulièrement le football, à la lumière d'une démarche de management de la chaîne d'approvisionnement, en s'interrogeant sur la manière dont se crée la valeur et dont elle est capturée par tel ou tel acteur. Son étude approfondie du championnat anglais souligne ainsi que ce sont les principaux fournisseurs des clubs, à savoir les joueurs, qui ont été capables de capter ladite valeur à travers une inflation continue des salaires des stars.

Or, les business models européen et nord-américain adoptent à ce niveau des modes opératoires très différents. Si le business model européen se fonde sur une " chaîne d'approvisionnement ", pour reprendre Lonsdale (2004), qui privilégie comme unité d'œuvre le club, le business model nord-américain privilégie comme unité d'œuvre la ligue fermée dans sa globalité. En d'autres termes, la stratégie d'achat de clubs comme Barcelone ou Manchester United est de capturer les meilleurs joueurs pour asseoir leur position dans la hiérarchie européenne des clubs, seule manière de garantir un flux continu de revenus associés à la vente d'une image de marque. En revanche, pour les ligues fermées nord-américaines, il s'agit de collectiviser la stratégie d'achat en vue de permettre une répartition harmonieuse et équitable des meilleurs joueurs, y compris dans les plus faibles clubs à la fin d'une saison, pour garantir un rééquilibrage des forces inducteur d'incertitude sportive. Imaginer en Amérique du Nord qu'une même équipe remporte sept fois d'affilée un championnat national, comme cela fut le cas de l'Olympique Lyonnais dans les années 2000, autrement dit multiplie les sweeps (séries gagnées sans perdre de match), est tout simplement inconcevable. Face à de nombreuses voix qui, en Europe, s'élèvent pour pousser à la création d'une Champions League fermée, assistera-t-on dans les prochaines années à une coexistence durable ou, au contraire, à terme, au triomphe du business model nord-américain ?

\section{Le modèle européen}

D'une manière simplificatrice, I'histoire du football professionnel européen peut être analysée à partir du rapport salarial noué entre le joueur et son club. Un footballeur professionnel représente un capital humain spécifique, fondé sur ses compétences, son habileté, sa capacité à s'intégrer (ou non) dans un collectif de joueurs. Comme tout individu impliqué dans le fonctionnement d'une organisation, il dispose de capacités productives fondées sur l'accumulation de connaissances générales ou spécifiques, de savoirfaire, etc., ce qui rejoint l'approche théorique initiée par Becker (1964). En bref, le footballeur professionnel dispose d'un savoir-faire en partie inné, en partie acquis grâce à la formation reçue. La question est de savoir comment les joueurs ont réussi, au fil du temps, à valoriser ce capital humain à leur avantage. Ainsi, dès la fin des années 1960, Sloane (1969) sera le premier chercheur à signaler que les performances sportives d'un club de football découlent des capacités de ses joueurs à proposer une prestation de qualité. Même si la notion de capital humain n'est pas explicitement revendiquée, elle constitue le soubassement théorique de la contribution.

Dans le cas européen, trois âges peuvent être distingués, qui correspondent à trois registres singuliers de valorisation du capital humain : (1) un âge préprofessionnel de liberté de mutation ; (2) un âge d'encadrement total par le biais du contrat à vie ; (3) un âge de libéralisation suite à la mise en place d'un contrat à durée déterminée (Gouguet et Primault 2002). Le passage du contrat à vie au contrat à durée limitée marque une transformation radicale du secteur du football professionnel. En effet, avec le contrat à vie, un joueur professionnel se trouve contraint de signer un engagement qui le lie à un club pratiquement pour toute sa carrière. C'est le club qui, de manière unilatérale, gère ce joueur au gré d'objectifs sportifs et/ou financiers. Wahl et Lanfranchi (1995), 
reprenant les mots du célèbre Raymond Kopa, n'hésitent pas à parler d'une période d'esclavage où, quelle que soit la volonté personnelle du joueur, ce dernier n'est jamais maître de son destin (voir Encadré 1) ; Taylor (2016) reste toutefois nuancé dans son jugement, en soulignant la présence de relations plus complexes entre les propriétaires de clubs et leurs salariés, et de marges de manœuvre aux mains de ces derniers. II n'en reste pas moins que l'on peut ici parler d'atteinte à la liberté du travail dans un contexte défavorable aux joueurs, notamment faute d'actions collectives (syndicales) en vue de défendre leurs intérêts. Dans leur ouvrage, Faure et Suaud (1999) rappellent les nombreux exemples de brillantes carrières brisées par des propriétaires de clubs refusant le transfert de tel ou tel joueur dans un club prestigieux ou, pire encore, le transfert obligé de joueurs talentueux (mais "réfractaires ») dans des clubs mineurs, pour les punir de leurs velléités de remise en cause de l'ordre établi.

La situation devient progressivement explosive au début des Trente Glorieuses, ainsi qu'en témoigne la multiplication d'actions collectives, tout au long des années 1960, pour faire reconnaître une liberté minimale de travail aux joueurs professionnels. Ces luttes aboutissent à la formulation d'un contrat d'achat d'un nouveau type, à durée déterminée, selon lequel est enfin reconnu le droit des joueurs à s'engager pour une période contractuellement négociée avec les propriétaires d'un club (voir Encadré 1). II résulte des nouvelles règles du jeu une volatilité accrue de la ressource humaine dans le cadre d'un marché du travail qui tend à se dualiser, avec la présence simultanée d'un marché primaire de joueurs à fort potentiel, dont le capital humain est élevé, et un marché secondaire de joueurs plus aisément interchangeables, dont le capital humain est moyen ou faible (Drut 2014). Certes, l'atteinte de résultats sportifs de bon niveau nécessite l'association de joueurs complémentaires dont certains

Encadré 1. La fin programmée du contrat à vie.

Le sport professionnel a été institué en 1932 en France mais, jusqu'à la fin des années 1960, les footballeurs disposent d'un statut extrêmement précaire que Raymond Kopa associe à de "l'esclavage » en 1963. Cette sortie médiatique fracassante lui vaut alors six mois de suspension. À cette époque, l'athlète signe avec le club un contrat "à vie ", jusqu'à l'âge de 35 ans. Le footballeur devient la propriété du club qui peut le céder à sa guise en obtenant une indemnité sans que le joueur ait véritablement son mot à dire. Ce système, qui s'apparente à un vaste marché aux bestiaux, finit par craquer sous la pression notamment de l'Union syndicale des footballeurs (UNFP) fondée en 1961. Un premier pas est fait en 1964 avec la création d'un régime de prévoyance permettant aux joueurs d'obtenir un pécule au terme de leur carrière. Après les événements de mai 1968, le contrat à vie est aboli. La France devient le premier pays au monde à mettre en place un contrat " à temps ", à durée limitée et variable, pour les joueurs professionnels, en juillet 1969.

Source : D'après http://sport24.lefigaro.fr/, 28 avril 2015. ne seront jamais des stars, mais la segmentation du marché du travail devient une réalité parfois cruelle, notamment lorsque des joueurs vieillissants passent du marché primaire au marché secondaire. La volatilité précédemment évoquée est surtout le fait de joueurs du marché primaire, aux compétences et à I'habileté plus affirmées et qui représentent environ $20 \%$ du nombre total de joueurs (Gouguet et Primault 2002). Ces joueurs, dont la valeur économique augmente parfois rapidement suite à une surexposition médiatique, auront à l'évidence tout intérêt à négocier des contrats de plus en plus courts et y faire intégrer des clauses précises de renégociation salariale.

La suppression des entraves à la mobilité ne rend ainsi plus vraiment étonnant qu'un joueur revendique le passage par six ou sept clubs lors de sa (brève) carrière. L'accusation la plus courante relative à cette mobilité exacerbée porte sur les agents de joueurs, dont le métier est de mettre en rapport contre rémunération vendeurs (joueurs) et acheteurs (clubs). L'agent étant rémunéré par des commissions lors de chaque transfert, son intérêt évident est d'en multiplier le nombre, notamment en jouant sur le capital relationnel construit dans un milieu finalement très fermé (Demazière et Jouvenet 2011). II faut néanmoins aussi reconnaître une responsabilité particulière aux propriétaires de clubs dans cette multiplication des carrières nomades (Paché et N'Goala 2011). Dès l'instant où leur objectif est d'accroître la valeur économique des joueurs appartenant au marché primaire, ils ne font qu'exacerber la mobilité des meilleurs d'entre eux, sachant que les transferts de club à club en cours de contrat génèrent un flux additionnel de recettes tant pour le club (compensation financière pour rupture anticipée de contrat) que pour ces joueurs (augmentation significative du salaire et des avantages associés). Comme le note Schotté (2016), la logique d'action qui en émerge est ainsi celle d'un joueur pensé comme un bien économique que des clubs s'échangent dans une perspective autant sportive (pour renforcer l'effectif) que financière (pour générer des recettes fatales additionnelles).

Le Tableau 1 indique le Top 10 des joueurs de football qui ont le plus généré de revenus liés à une rupture anticipée de contrat, parfois après un passage-éclair dans leur club. C'est notamment le cas d'Angel Di Maria, qui n'est resté présent dans l'effectif de Manchester United qu'une seule saison, avant d'être racheté en août 2015 par le Paris SaintGermain pour 63 millions d'euros. Bien évidemment, il ne faut en tirer aucune conclusion hâtive quant à l'existence d'un modèle universaliste de stratégie d'achat générant un nomadisme généralisé des stars. Certains clubs préfèrent au contraire identifier très tôt de jeunes talents prometteurs et les conserver le plus longtemps possible dans leur effectif, à l'image de 
Tableau 1. Top 10 des joueurs ayant généré les plus importants montants de transferts cumulés.

\begin{tabular}{lcc}
\hline Joueurs & Nombre de transferts & $\begin{array}{c}\text { Transferts cumulés } \\
\text { (millions d'euros) }\end{array}$ \\
\hline Angel Di Maria & 4 & 180 \\
Zlatan Ibrahimovic & 7 & 169 \\
James Rodriguez & 4 & 132,6 \\
Nicolas Anelka & 8 & 127,4 \\
Hernàn Crespo & 4 & 119,3 \\
Juan Sebastián Verón & 6 & 116 \\
Luis Suàrez & 4 & 115,8 \\
Falcao & 4 & 113 \\
Cristiano Ronaldo & 2 & 111,5 \\
Gareth Bale & 2 & 108,7 \\
\hline Source : D'après http://www.transfermarkt.fr/ (consulté le 31 mai 2016)
\end{tabular}

Lionel Messi ayant effectué toute sa carrière professionnelle à Barcelone, en parfaite osmose avec son mentor Andrés Iniesta, avec lequel il a tissé un lien filial indéfectible (Brach 2012). II n'en reste pas moins que les revenus tirés de ruptures anticipées de contrat participent à la puissance financière d'un club, parallèlement aux recettes hors guichet qu'il engrange (merchandising, droits TV), et contribuent directement à sa suprématie sportive. Les analyses économétriques conduites par Marquès (2015) sur le football professionnel européen et par Llorca et Teste (2016) sur la Ligue 1 française, couvrant dans les deux cas une période de dix ans, indiquent d'ailleurs une relation étroite et positive entre les moyens financiers à la disposition d'un club et ses résultats sportifs.

Un tel accroissement du montant cumulé des transferts ne peut cependant se produire qu'à la condition que la durée des contrats originels s'allonge, permettant au capital humain de rapidement gagner en valeur en dégageant à la revente une plus-value significative. En d'autres termes, il s'agit de disposer devant soi de plusieurs années afin d'accroître la valeur marchande d'un joueur talentueux, puis d'accepter son départ bien avant la fin du contrat contre une indemnité pour rupture anticipée. Dans cette logique de marchandisation, la captation du capital humain à fort potentiel réclame des ressources financières de plus en plus importantes que seule une poignée de clubs européens possèdent. Dans son édition du 2 septembre 2015, LeMonde.fr note ainsi que les 50 transferts les plus onéreux de l'histoire du football professionnel européen se concentrent sur quelques clubs, sachant que seulement huit grandes formations monopolisant deux tiers des achats : Barcelone, Liverpool, Chelsea, Lazio de Rome, Manchester City, Manchester United, Paris Saint-Germain et Real Madrid. La hiérarchie sportive entre clubs finit par être le reflet de la hiérarchie financière, comme en témoigne la présence systématique d'une douzaine de clubs en compétition frontale, toujours les mêmes, lors des phases finales de la Champions League européenne depuis une décennie (des Garets, Nicholson et Paché 2016). Un tel constat converge d'ailleurs avec l'analyse de Meyssonnier et Mincheneau (2013), pour qui la réalité du football européen est celle d'une poignée de grandes équipes capables de se doter d'un système de pilotage global favorisant pérennité managériale et performances sportives récurrentes.

\section{Le modèle nord-américain}

La réalité est toute autre dans le sport professionnel en Amérique du Nord. La ligue fermée y constitue l'unité économique de référence fondée sur une pratique volontaire de cartellisation, qui conduit même à une politique marketing commune aux différents clubs, y compris en termes de ventes de tickets pour les rencontres (Marquès et de Bontin 2014). Les membres de la ligue poursuivent en fait une stratégie collective pour élaborer et vendre un produit attractif, et en extraire la rente induite (Hoehn et Szymanski 1999). Les éventuelles restructurations sont organisées par la ligue elle-même, qui définit par ailleurs des modes de régulation sophistiqués au niveau des achats des joueurs les plus talentueux afin d'équilibrer les forces en présence et d'éviter la domination outrageuse et durable d'un club. II s'agit de la fameuse draft dont les mécanismes sont synthétisés par Bourg et Gouguet $(2012,63)$ : « La draft octroie aux clubs des droits exclusifs sur les nouveaux joueurs intégrant la ligue et issus des championnats universitaires, des ligues mineures ou de ligues étrangères. Le dispositif vise à rééquilibrer sportivement la compétition mais également à limiter la concurrence entre les clubs. Le choix se fait dans l'ordre inverse du classement de la dernière saison. » En d'autres termes, les clubs les plus mal classés en fin de saison disposent d'une sorte de droit de préemption leur permettant de recruter les talents les plus prometteurs, pour espérer briller la saison suivante et surprendre les spectateurs par des performances inattendues.

Nous nous retrouvons incontestablement ici dans une posture d'interdépendance mutuelle qui régit la gouvernance de la ligue (Sloane 2015). La logique symbiotique associant des clubs d'une ligue fermée ne pouvant vivre les uns sans les autres est d'ailleurs confortée par la règle du salary cap, autrement dit le plafonnement imposé de la masse salariale globale des joueurs de la ligue ; il implique une négociation collective, souvent âpre, entre joueurs et propriétaires de clubs aux objectifs divergents. À la différence du football professionnel européen, il est ainsi hors de question que les clubs les plus riches assèchent le marché des stars et produisent ainsi une distorsion durable des conditions de la concurrence sportive, au final nuisible pour le caractère « médiatique » de la ligue. Cette stratégie de " contrôle » des joueurs est l'objet de nombreux débats, notamment sur le plan juridique (Graves 1998), mais elle témoigne d'une volonté de mutualisation des revenus au bénéfice de tous les acteurs de l'industrie (la ligue), qui se poursuit par une vente collective des droits de retransmission télévisée, essentiels à la survie de toute ligue. La présence d'un capital humain se vérifie évidemment dans le modèle des ligues nord- 
américaines, mais davantage dans le cadre d'une stratégie concertée d'achats de joueurs, en fonction des intérêts du collectif de clubs (la franchise), plutôt qu'en fonction uniquement de celle de chacun des clubs.

En Amérique du Nord, il y a évidemment plusieurs ligues professionnelles; quelques-uns des meilleurs joueurs y gagnent environ l'équivalent annuel de 7 millions d'euros. Comme il existe des différences entre les ligues de basketball, de baseball, de football (soccer) et de hockey, nous n'allons retenir que l'une de celles-ci à titre d'illustration, à savoir la Ligue Nationale de Hockey $(\mathrm{LNH})$. Bien que cette ligue existe depuis un peu plus d'une centaine d'années, les changements apportés pour rendre les équipes de plus en plus compétitives sont particulièrement intéressants. On peut synthétiser de la manière suivante les caractéristiques les plus pertinentes concernant la stratégie d'achat de joueurs (gestion du capital humain et organisation collective du marché du travail) et la fixation d'un plafond salarial :

- Une gestion coercitive du capital humain. Les joueurs dont le contrat échoit (c'est le cas au 30 juin pour tous les contrats, car ils sont à date fixe) deviennent « agents libres » et peuvent alors en venir à un accord avec toute autre équipe. C'est vers la fin des années 1960, pratiquement au même moment où s'opère la dérèglementation du football européen, qu'un premier joueur (de baseball) va exercer de multiples actions afin de choisir librement l'équipe pour laquelle il allait évoluer, le tout visant évidemment à obtenir un meilleur salaire. Suite à différents recours juridiques et arrêts de travail, cette approche est devenue une réalité au cours des décennies suivantes dans toutes les ligues professionnelles en Amérique du Nord. Le système mis en place contourne cette liberté en adoptant la draft, mais dépouillée des risques opportunistes. En effet, l'équipe terminant à la dernière place choisissait en premier ; certaines équipes perdaient presque volontairement les ultimes matches pour s'assurer de terminer dernier! D'où la mise en place d'un système complexe de "loterie » permettant, avec des probabilités décroissantes, aux cinq équipes ayant obtenu les moins bons résultats, d'avoir la " chance » de choisir en premier lieu.

- Une organisation collective du marché du travail. La date limite des transactions est programmée cinq semaines environ avant la fin de la saison régulière (avant les séries éliminatoires). Certaines équipes ayant une probabilité faible d'être dans ces séries sont portées à échanger certains joueurs, entre autres pour une meilleure position au repêchage («draft » en anglais). Concrètement, un joueur est repêché par l'une des équipes, lors du repêchage annuel des joueurs, et doit impérativement s'y présenter. Le contrat habituellement signé est de trois ans, à raison d'environ 950000 dollars US au maximum par an, quoique des clauses de bonus puissent être additionnées. Le défi d'une équipe est surtout de choisir dans les différentes rondes de repêchage les joueurs qui pourraient faire carrière, et qui sont donc " sous-estimés » lors de leur évaluation par la ligue et les autres équipes.

- Le plafond salarial. Il s'agit de la règle du salary cap, renégociée par la $\mathrm{LNH}$ année après année : de 39 millions de dollars US en 2005-2006, le plafond salarial a bondi à 71 millions de dollars US en 2015-2016, sachant que c'est le salaire moyen d'un joueur qui est comptabilisé pour calculer ledit plafond salarial. Ce dernier est établi à partir du pourcentage des revenus de la LNH ; plus la ligue a un chiffre d'affaires élevé, plus le pourcentage remis aux joueurs augmente (quoique de $1 \%$ à la fois...). II existe également un plancher salarial, fixé à 52,8 millions de dollars US en 2015-2016, afin d'assurer un certain niveau de revenus aux joueurs, et d'éviter qu'une équipe n'attire que de moins bons joueurs et se retrouve, pendant un certain temps, avec plusieurs choix de repêchage particulièrement bien placés (il est reconnu qu'il y a une grande différence entre les dix premiers choix au repêchage annuel et les suivants). Ces planchers et plafonds valent pour les 23 joueurs réguliers d'une formation. En France, la Ligue Nationale de Rugby (LNR) a introduit un salary cap depuis 2010, mais il est loin de faire l'unanimité (voir Encadré 2).

II est clair que des efforts continus sont faits par la LNH et, d'une manière générale, par les ligues fermées, pour un meilleur équilibre collectif entre les équipes, et ceci a un impact évident sur la valeur de la franchise. Celle-ci est largement fonction des droits TV, eux-mêmes dépendant des taux d'écoute et, par conséquent, de l'intérêt de regarder des matchs âprement disputés par des stars. Le droit d'entrée pour rejoindre une franchise sera alors d'autant plus élevé que son attractivité, fondée sur des mécanismes de mutualisation des ressources et de gestion

Encadré 2. Le salary cap, une culture purement nordaméricaine?

Né aux États-Unis, et unanimement appliqué par les grandes ligues telles que la NBA (basketball), la NFL (football américain) ou encore la NHL (hockey), le salary cap consiste à plafonner la masse salariale des clubs. II s'agit de préserver une équité sportive en permettant une concurrence effective entre les clubs, tout en les prémunissant d'un éventuel endettement abyssal. Unanime outreAtlantique, le salary cap reste pour le moment anecdotique en Europe. Unique ligue sportive française à avoir instauré le salary cap, Ia Ligue Nationale de Rugby (LNR) limite depuis 2010 les clubs de son élite, le Top 14, à une masse salariale plafonnée à 10 millions d'euros par saison sportive (contre, à titre d'exemple, plus de 63 millions de dollars en NBA et plus de 130 millions de dollars en NFL). Après près de quatre années d'expérience, le dispositif demeure critiqué et il est loin de faire l'unanimité. Sa légalité est même questionnée (atteinte au principe communautaire fondamental de libre circulation des travailleurs, remise en question du principe de libre concurrence, etc.).

Source : D’après http://www.juritravail.com/ (consulté le 16 avril 2016). 
Tableau 2. Quelques exemples de mutualisation logistique en France.

\begin{tabular}{|c|c|c|c|}
\hline Région & Types de produits & Service et modalités de la mutualisation & Acteurs \\
\hline Poitou-Charentes & Agro-alimentaire & Service de commande unique du magasin vers les TPE-PME via un portail & PME, distributeurs, ARIA \\
\hline Toutes régions & Vin & $\begin{array}{l}\text { Acheminement groupé des sites viticulteurs fournisseurs vers les magasins } \\
\text { Auchan }\end{array}$ & $\begin{array}{l}\text { Auchan France, } \\
\text { viticulteurs régionaux }\end{array}$ \\
\hline Normandie & Agro-alimentaire & Acheminements groupés de commandes vers les restaurants scolaires & $\begin{array}{l}\text { Industriels, restaurateurs } \\
\text { scolaires }\end{array}$ \\
\hline Normandie & Agro-alimentaire & $\begin{array}{l}\text { Service de mise en relation entre producteurs et transporteurs en vue d'un } \\
\text { regroupement des livraisons clients }\end{array}$ & $\begin{array}{l}36 \text { industriels, } 140 \\
\text { transporteurs }\end{array}$ \\
\hline PACA & Alimentaire & Distribution groupée sur des plates-formes de cross docking de Carrefour & $\begin{array}{l}\text { Campbell, Haribo et } \\
\text { Carrefour }\end{array}$ \\
\hline Champagne-Ardenne & Agro-alimentaire & Partage de l'outil logistique en matière de stockage et de transport & 6 industriels \\
\hline
\end{tabular}

Source : D'après http://www.aslog.org/ (consulté le 29 dècembre 2015).

salariale, est elle-même forte. La LNH a ainsi programmé une expansion pour la saison 2017-2018, et le milliardaire Bill Foley, propriétaire de la nouvelle équipe des Golden Knights de Las Vegas, a dû débourser, en juin 2016, un peu plus de 500 millions de dollars US pour devenir le $31^{\mathrm{e}}$ franchisé d'un circuit en constante progression. En bref, joueurs, équipes et ligue sortent tous gagnants ! Ceci n'empêche pas certaines équipes de valoir davantage que d'autres : l'équipe de Toronto est ainsi évaluée à plus de 1,3 milliard de dollars US, alors que l'équipe valant le moins dans la LNH est évaluée à 160 millions de dollars US seulement ${ }^{1}$.

\section{La mutualisation comme grille de lecture}

La question de la mutualisation des ressources et des achats de joueurs caractérise les stratégies collectives inhérentes au fonctionnement de la LNH. Elle apparaît comme un élément central pour augmenter collectivement les revenus de cette ligue. Pour mieux appréhender ce phénomène, revenons un instant sur les travaux menés sur la mutualisation dans le domaine du management, tout particulièrement en management stratégique et logistique des relations inter-organisationnelles. La mutualisation, admise comme la volonté d'acteurs indépendants de partager des ressources en vue d'un usage plus efficient et efficace, n'est certes pas un phénomène nouveau. Depuis le milieu des années 1980, le principe de mutualité a accompagné la mise en œuvre des stratégies de coopération interentreprises et des structures en réseau qui en découlent. Il a été alors question de mutualisation verticale, c'est-à-dire de mise en commun de moyens, de ressources et de compétences complémentaires, autour d'un projet productif unissant des entreprises sur une même chaîne logistique. De nombreux cas d'entreprises dans l'automobile (Toyota, MCCSmart), l'informatique (Dell, IBM), l'aéronautique (Airbus, Boeing, Dassault Aviation) ou le textile (Benetton, Golden Lady) illustrent ces démarches stratégiques. Le tableau 2 fournit un certain nombre d'exemples de mutualisation verticale dans le contexte français, une pratique particulièrement vivace dans plusieurs filières agro-alimentaires, et dont l'initiateur peut autant être un industriel, un grand distributeur ou une institution publique.
Parallèlement, des mutualisations de moyens ont progressivement vu le jour entre des entreprises exerçant le même métier. De telles mutualisations entre concurrents directs sont alors qualifiées de mutualisations horizontales et vont généralement de pair avec des stratégies de coopétition (Brandenburger et Nalebuff 1996). Les démarches observées dans la LNH se rapprochent davantage de ce type de mutualisation qui, depuis plus de vingt ans, a largement été étudiée en logistique et supply chain management (voir par exemple Camman et al. [2013], Senkel, Durand et Hoa Vo [2013] et Montoya-Torres, Muñoz-Villamizar et Vega-Mejía [2016]). Principalement observée dans le transport, notamment avec l'essor de l'industrie de la prestation logistique que l'on retrouve bien souvent au cœur des pratiques de gestion mutualisée des approvisionnements (GMA) (Abbad 2014), cette mutualisation résulte de collaborations horizontales visant principalement à regrouper des moyens dans le but de massifier les flux physiques. Cela s'effectue généralement sur le site d'un prestataire de services logistiques qui prend en charge l'organisation des tournées de livraison à destination des distributeurs. De manière peut-être surprenante, mais finalement moins qu'il n'y paraît, l'agent sportif précédemment évoqué ne pourrait-il pas jouer le rôle d'acteur tiers au sein des chaînes d'approvisionnement décrites par Lonsdale (2004) dans le cadre du football européen, au même titre que les prestataires de services logistiques dans la commercialisation des produits de grande consommation?

Aujourd'hui, les évolutions constantes de l'environnement économique semblent conforter le recours à la mutualisation horizontale. Tel est le choix de cinq géants et concurrents mondiaux de l'hygiène et de la cosmétique (Colgate, Henkel, GSK, Sara Lee ainsi qu'Eugène Perma depuis 2011) qui, par une démarche de pooling initiée par le prestataire FM Logistic, mutualisent leurs expéditions vers la grande distribution. L'objectif affiché est de saturer les moyens de transport, d'optimiser les livraisons et, globalement, réduire les coûts tout en augmentant les fréquences de livraison et la qualité de la prestation. Mais la mutualisation horizontale intéresse également des PME régionales qui, en s'associant pour acheter ensemble des services logistiques, 
sont capables de réduire significativement leurs coûts de distribution, comme la recherche de terrain conduite par Ghaderi et al. (2012) en témoigne. En France, la création du GIE Chargeurs Pointe de Bretagne a pour objectif de rassembler des entreprises bretonnes que l'éloignement des marchés oblige à collaborer pour faire face à l'augmentation induite des coûts de transport. Une plateforme de consolidation et des boucles de ramasse parfaitement organisées permettent ainsi une maximisation du taux de remplissage des véhicules de transport sur les différentes lignes d'approvisionnement (voir la Figure 1).

Cependant, des collaborations tant verticales qu'horizontales échouent, d'autres n'apportent pas les résultats escomptés. $Y$ aurait-il des facteurs clés de succès de la mutualisation à identifier ? Ces facteurs pourraient-ils donner une nouvelle lecture des stratégies de mutualisation des ressources pour renforcer la position des ligues fermées, dont la LNH ? Si, dans les travaux sur les collaborations verticales, la question de l'exercice du pouvoir entre les acteurs est récurrente (voir par exemple Maloni et Benton [2000] et Cox [2001]), ceux portant sur les collaborations horizontales lui accordent moins d'importance. Dans ce second cas de figure, il est davantage souligné la quasi égale répartition des pouvoirs entre les partenaires en coopétition, ce qui explique l'absence d'acteur pivot dans de telles structures en réseau, et l'importance de s'assurer de la présence d'une bonne ouverture d'esprit, d'une recherche permanente de compromis et du réel engagement dans le processus de mutualisation des différents acteurs impliqués. Tel est le cas en matière d'achat de prestations logistiques auprès d'acteurs spécialisés pour la collaboration horizontale entre Colgate, Henkel, GSK, Sara Lee et Eugène Perma précédemment évoquée.

La recherche conduite par Montalan et Vincent (2011) sur la mutualisation des achats hospitaliers a pour vertu de souligner combien la démarche reste difficile à initier, tant au plan organisationnel qu'au plan opérationnel. Au plan organisationnel, les acteurs ont parfois du mal à se projeter dans un processus inter-organisationnel qui limite forcément leur latitude décisionnelle. Au plan opérationnel, mutualiser les achats entre plusieurs hôpitaux suppose que les utilisateurs expriment leurs besoins en termes de fonctionnalités attendues, et pas en termes de produits attendus, sachant que la stratégie collective ne peut en aucun cas se réduire à la somme de stratégies individuelles. Or, les ligues nord-américaines de sport professionnel, dont la $\mathrm{LNH}$, ont su historiquement surmonter ces difficultés. Dès la création du sport professionnel en Amérique du Nord, à la différence de l'Europe, l'unité de référence a été la ligue et sa rentabilité, et non pas le club en tant que tel; se projeter dans un processus inter-organisationnel est donc «génétiquement 》 ancré dans les modes opératoires du business model. Quant aux dimensions opérationnelles, un total consensus existe sur la fonctionnalité de la mutualisation des achats de joueurs, à savoir maintenir l'attractivité de la ligue auprès des médias par le biais d'une stratégie collective d'actions concertées.

La force des ligues nord-américaines est ainsi d'avoir compris et intégré le fait qu'il faut à tout prix éviter que quelques équipes captent systématiquement les

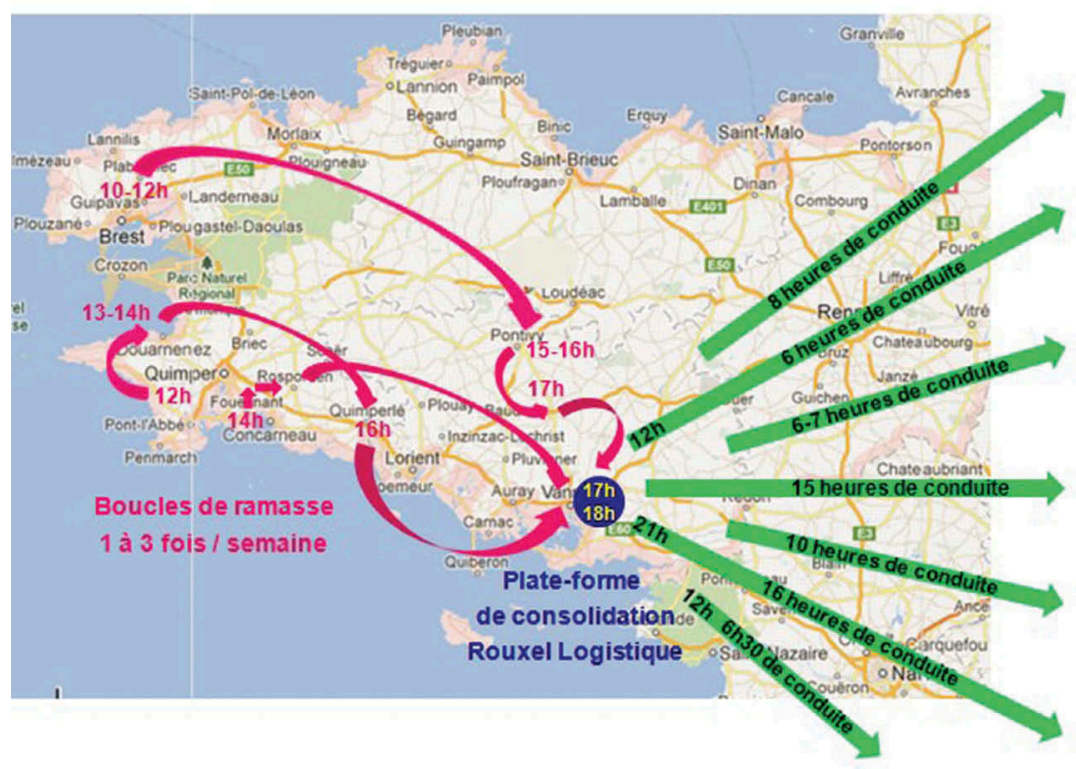

Carrefour Bassens : A/C 5h Auchan Blanquefort: AVD 13h
15 tractions

1 à 3 fois / semaine

Carrefour Brebières: AVC $\mathbf{5 h}$

Auchan Sainghin : AVD $7 \mathrm{~h}$

Auchan Amiens :

Atac Amiens

Carrefour Thuit-Hébert: AVC $\mathbf{8 h}$

Carrefour Le Mans : AVC 2h

Carrefour Dugny : AVC 4h

Carrefour Moissy : AVC $6 \mathrm{~h}$

Auchan Epône

Auchan Duttlenheim : AVD 9h30

Atac Strasbourg : 11h

Carrefour Pont de Veyle : AV 5h

Carrefour Brignoles : AVD 5h

Carrefour Agen : AVC

Figure 1. Un exemple de boucles de ramasses et de livraisons au sein du GIE Chargeurs Pointe de Bretagne.

Source : Document interne du GIE Chargeurs Pointe de Bretagne (2015). 
meilleures ressources et soient toujours présentes lors des phases éliminatoires, à la différence de la Champions League européenne. Si cela était le cas, il pourrait en effet s'ensuivre une lassitude des spectateurs face à l'ennuyeuse " certitude du résultat ", et finalement une perte de valeur du produit que propose la ligue, engendrant inévitablement une baisse des droits TV ... et des revenus de chaque club franchisé. Comme on le voit, dans le cas des ligues de sports professionnels en Amérique du Nord, la force de la mutualisation des achats de joueurs réside justement dans la création d'une valeur collective pour toutes les entreprises, à savoir les clubs franchisés, qui se coordonnent dans une perspective inter-organisationnelle afin d'augmenter la rente créée. Mais quels sont réellement les facteurs clés de succès de ces ligues fermées ? Sur quels principes d'achat de joueurs repose leur succès ? Existe-t-il différentes stratégies d'achat possibles sur un marché qui se veut de plus en plus internationalisé ? La section suivante fournit quelques éléments de réflexion pour aborder ces questions complexes.

\section{Discussion et conclusion}

Le domaine sportif peut être considéré sous plusieurs angles ; évidemment, il y a celui des résultats sportifs obtenus par les clubs, mais aussi ceux des stratégies de jeu utilisées lors des matchs, de l'approche économique liée au financement de l'activité, ou encore du brand marketing. Parfois même, l'artiste se laisse emporter pour décrire une passion dévorante, à l'image de Toussaint $(2015,11)$ : « Le football, comme la peinture, selon Léonard de Vinci, est cosa mentale, c'est dans l'imaginaire qu'il se mesure et s'apprécie. » Le présent article a choisi d'aborder cette réalité de façon originale, sous l'angle singulier du management des achats, plus spécifiquement en regardant comment la notion de mutualisation peut être utile pour mieux comprendre les avantages découlant de l'approche collective des ligues nord-américaines en matière de politique d'achat de joueurs et, plus largement, de gestion du capital humain. Nul doute qu'une telle approche peut surprendre au premier abord les tenants d'une vision étroite des questions liées au management des achats. Elle éclaire pourtant d'un jour nouveau les enjeux de stratégies collectives originales, qui participent à une proposition de valeur satisfaisant à la fois les clients finaux, les annonceurs et les chaînes de télévision (Marquès et de Bontin 2014).

L'étude comparative des championnats européens et des ligues nord-américaines fait ainsi émerger deux logiques d'action différentes : la compétition pour les premiers, la coopération pour les secondes. Marquès (2011) modère toutefois le propos en ce qui concerne le football européen. En effet, selon lui, le fait que des clubs professionnels de football puissent, à un moment donné, prêter certains de leurs joueurs à des clubs avec lesquels ils sont en concurrence directe dans un (ou des) championnat(s) renvoie à un registre plutôt de nature coopétitive. Si l'idée semble séduisante, il n'est toutefois pas possible ici de parler de stratégie collective de mutualisation horizontale dans la mesure où les joueurs ponctuellement mis à disposition d'un autre club ne le sont pas sur la base d'un capital humain à fort potentiel, comme c'est le cas dans la draft nord-américaine, mais parce qu'ils ne s'intègrent pas (ou plus) dans le système de jeu initié par l'entraîneur, quitte à renforcer un concurrent direct et à « éclater » sur le terrain contre toute attente. Au demeurant, il n'est pas rare de voir un joueur prêté pour un temps réintégrer son équipe d'origine après un changement d'entraîneur, et il est encore plus rare (voire inimaginable) de penser qu'un club prête l'une de ses stars en cours de saison!

Adoptant une perspective institutionnelle, Andreff (2007) va encore plus loin en introduisant l'idée de deux modèles singuliers de régulation dont le tableau 3 précise les principales caractéristiques, en n'hésitant pas à parler, en forme de clin d'œil, d'une régulation « quasi-socialiste » des ligues fermées au cœur même du capitalisme américain. Le dialogue social est d'ailleurs un élément clé du fonctionnement des ligues fermées en vue d'éviter les situations de lock out, qui ont touché à plusieurs reprises la $\mathrm{LNH}$, mais aussi la fameuse NBA. Le lock out correspond à la fermeture provisoire d'une ligue professionnelle, suite à un désaccord majeur entre les propriétaires des équipes et les joueurs (Vrooman 1995). Alors que les propriétaires cherchent à contrôler la progression des salaires et ralentir une inflation des coûts, les joueurs, réunis sous l'égide de syndicats, cherchent à maximiser la part des revenus qu'ils reçoivent de la ligue. Le dialogue social est ici la clé d'une volonté d'échapper à une " destruction collective de valeur »,

Tableau 3. Deux choix institutionnels singuliers opposant l'Europe à l'Amérique du Nord.

\begin{tabular}{lll}
\hline Institutions & \multicolumn{1}{c}{ Choix nord-américains } & \multicolumn{1}{c}{ Choix européens } \\
\hline Ligue & Fermée & Ouverte \\
Entreprises (clubs) & Maximisation du profit & Maximisation des victoires \\
Mobilité des clubs & Horizontale: délocalisation possible de la franchise & Verticale: système de relégation et de promotion \\
Marché du travail & Très régulé & Dérégulé (depuis 1995) \\
Nouveaux financements & Naming, merchandising & Droits TV, cotation en bourse \\
Concurrence & Exemption de la loi antitrust, monopole /monopsone de & Politique de concurrence européenne, pourvoir de marché des \\
& la ligue & grands clubs \\
\hline Source: D'après Andreff (2007)
\end{tabular}

Source : D'après Andreff (2007). 
conduisant le syndicat des joueurs et la ligue à signer un accord pluriannuel dénommé collective bargaining agreement (Keefer 2016). Ceci ne correspond en rien au cadre juridique européen qui laisse libre cours au face à face direct entre le joueur (via son agent sportif) et son propriétaire.

La cartellisation qui caractérise les ligues nordaméricaines n'est évidemment pas sans impacts négatifs, notamment en termes de métropolisation et de domination d'un nombre réduit d'équipes (et donc de villes) sur le marché du sport professionnel en Amérique du Nord. La rentabilité d'une ligue fermée découle de la demande d'un public, et non d'une fédération sous l'autorité de la puissance publique. Cette demande va se concentrer naturellement autour de quelques pôles urbains, ce qui génère au final une discrimination spatiale exacerbée éliminant petit à petit des villes de taille moyenne de la ligue fermée (Helleu et Durand 2007). D'une certaine manière, nous retrouvons ici la problématique des places centrales de Christaller (1933/2009), qui place au cœur de son analyse la notion de centralité. La tendance identifiée est celle d'un regroupement en un même lieu de services destinés à la population dispersée dans la région (ou zone d'influence) entourant la place centrale, et qui génère alors un effet d'attraction irrépressible. II en résulte une hiérarchisation urbaine en fonction de la distance que le consommateur accepte de parcourir pour se procurer le service. Si l'on considère qu'un spectacle sportif proposé par une ligue fermée est un service marchand, une franchise localisée dans une place centrale disposera ainsi d'une capacité de captation de spectateurs sur plusieurs dizaines (centaines ?) de kilomètres, en empêchant toute intégration d'un nouveau franchisé dans sa zone d'influence. Rien d'étonnant dès lors que les ligues fermées soient toutes sous le joug d'équipes dominantes, le plus souvent issues des quelques grands " marchés », celui des mégapoles comme New York, Los Angeles ou Chicago.

D'un point de vue managérial, force est de reconnaître que le cas des ligues nord-américaines constitue cependant un remarquable exemple d'une mutualisation réussie au niveau des achats. La question de la mutualisation de ressources entre concurrents est largement débattue depuis près de dix ans dans la littérature, mais il s'avère finalement difficile de trouver des exemples marquants de succès. Les ligues nordaméricaines en font incontestablement partie. En effet, quand on aborde une ligue sportive professionnelle à l'européenne, chacune des équipes constitue une entité qui vise à maximiser ses profits au détriment des autres; le résultat obtenu pour la ligue est alors moins intéressant que par la mutualisation. Cette dernière peut être associée à la stratégie de " partenariat », une approche reconnue en achat, car elle permet tant au fournisseur (ici le joueur) qu'à l'acheteur (ici le club) d'atteindre leurs objectifs par une approche
" gagnant-gagnant ». En rendant les équipes plus compétitives par les choix de repêchage accordés aux équipes ayant le moins bien réussi, il est normal de s'attendre à ce qu'un meilleur équilibre émerge assez rapidement. Cependant, il faut aussi tenir compte de la gestion de l'équipe : certaines ont plus de succès que d'autres, même si elles ne bénéficient pas de bons choix au repêchage. Par exemple, au niveau de la LNH, l'équipe ayant remporté le trophée lors de trois des six dernières saisons a pour stratégie de repêchage de " faire le plein » d'un grand nombre de joueurs amateurs, dans l'espoir que certains d'entre eux causeront une surprise agréable. Une logique identique se retrouve en achat quand certaines entreprises choisissent d'acheter des stocks promotionnels, même si elles ne savent pas toujours ce qu'elles en feront.

Quelques autres principes d'achat sont à l'œuvre dans le fonctionnement des ligues nord-américaines. Dans les recherches en management, un accent significatif est mis sur les achats stratégiques, par exemple dans la matrice de Kraljic (1983), mais des politiques appropriées sont différentes (et importantes) pour les autres catégories, qui trouvent un écho évident dans l'achat des joueurs : achats à goulot d'étranglement (exemple : certains joueurs sont particulièrement bons en situation de désavantage numérique), et même achats courants (exemple : les joueurs de soutien peuvent contribuer aux résultats au moment opportun). De même, vu l'attrait qu'exercent les salaires faramineux de l'élite sportive, de plus en plus de jeunes visent à devenir des athlètes professionnels ; le bassin de "fournisseurs » (joueurs) est alors plus grand qu'il ne l'était auparavant. Enfin, toutes les équipes de la LNH font passer plusieurs tests physiques et psychologiques (ou, à tout le moins, des entrevues serrées) aux jeunes auxquels elles s'intéressent ; c'est comme la pré-qualification de certains fournisseurs, afin de ne pas avoir à déterminer la qualité des produits arrivant en usine. En abordant la situation quelque peu différemment, on pourrait également découvrir que certaines équipes de divisions moins élevées contribuent davantage à la "production » de bons joueurs que d'autres au fil du temps; peut-être vaut-il alors la peine de développer d'excellents rapports avec celles-ci ?

Un autre lien à signaler est celui avec le volet international des achats. Le hockey se pratique dans plusieurs pays et certaines formations de la LNH semblent avoir développé un appétit pour des joueurs originaires de tel ou tel pays ; d'ailleurs, ces formations s'assurent d'avoir des recruteurs allant plus fréquemment dans ces pays. En achat, la même approche est utilisée : on développe une compétence et des relations avec des gouvernements et des fournisseurs de certains pays plutôt que d'autres. Par ailleurs, des entreprises développent certains de leurs fournisseurs en les aidant à s'améliorer au niveau de leur système d'offre (van Weele 2014). C'est le cas dans la LNH, où chacune des 
équipes possède au moins une équipe de niveau inférieur où elle peut envoyer certains joueurs s'améliorer ou se remettre d'une blessure. Lorsqu'ils sont à nouveau productifs, ils peuvent être rappelés dans le " grand club» (et tous espèrent y rester, avec un contrat en poche...). Nul doute que l'exploration des ligues professionnelles nord-américaines devrait ainsi permettre de mieux comprendre le caractère universel de certaines démarches de management, et ouvrir des avenues de recherche très prometteuses. Une analyse comparative des deux modèles présentés doit ainsi se poursuivre afin de savoir si la régulation du marché des transferts, la définition sous contrainte des politiques salariales et la généralisation des mécanismes de cartellisation sont envisageables en Europe dans un contexte économique, social et sportif radicalement différent.

En bref, il est impossible de répondre pour l'instant à la question posée en fin d'introduction de l'article sur le devenir des deux business models, le modèle européen et le modèle nord-américain. II faut néanmoins reconnaître que ce dernier repose sur une dynamique financière et une maîtrise de l'incertitude qui n'est pas sans faire rêver les dirigeants de grands clubs européens de football. Ainsi, il n'apparaît pas réellement surprenant, aux yeux des spécialistes du ballon rond, qu'à l'automne 2016, des clubs scandinaves (Malmö en Suède, Rosenborg en Norvège, Copenhague au Danemark), néerlandais (Amsterdam, Eindhoven, Rotterdam), belges (Bruges, Anderlecht) et écossais (Celtic Glasgow, Glasgow Rangers) aient évoqué la création d'une mini-Ligue fermée d'Europe du Nord, qui leur ferait quitter leurs championnats respectifs et se partager de lucratifs (et stables) revenus que I'UEFA, à travers l'organisation de la Champions League, n'accorde qu'à une poignée de puissants clubs. Toute la question sera évidemment de savoir si de telles ligues fermées pourront réellement fonctionner en l'absence d'un système de mutualisation des achats de joueurs, en évitant ainsi qu'un petit nombre de clubs tire bénéfice de rentes de situation et nuise à la valeur du produit sportif vendu aux réseaux TV. Ce qui conduirait alors à reproduire les déséquilibres que les clubs d'une hypothétique mini-Ligue fermée d'Europe du Nord souhaitent justement combattre.

\section{Nota}

1. Informations tirées du lien Internet suivant: http://www. forbes.com/sites/mikeozanian/2014/11/25/the-mostvaluable-teams-in-the-nhl/ (consulté le 26 mai 2016).

\section{Déclaration}

Les auteurs confirment qu'il n'y a aucun conflit d'intérêt.

\section{Les auteurs}

François Fulconis est maître de conférences en Sciences de Gestion à I'Université d'Avignon et des Pays de Vaucluse. Ancien du CEFAG (FNEGE Paris), il est membre du Centre de Recherche sur le Transport et la Logistique (CRET-LOG). Ses recherches s'inscrivent dans le domaine du management stratégique et logistique des organisations. Elles traitent plus spécialement des stratégies de coopération entre entreprises, des structures en réseau et des interfaces inter-organisationnelles. Elles ont fait l'objet de nombreuses publications (revues, colloques et ouvrages) dont, en collaboration, La prestation logistique : origines, enjeux et perspectives, publié aux Editions Management \& Société.

Jean Nollet est professeur titulaire de Sciences de Gestion à HEC Montréal, au Canada. Auteur de très nombreux articles académiques publiés dans les meilleures revues internationales, et de plusieurs ouvrages primés, titulaire de la Chaire de gestion des approvisionnements, il a fait des achats son domaine de prédilection pour l'ensemble de sa carrière. Son approche pratique lui a valu d'être invité à faire de nombreuses présentations aux professionnels du domaine et il a œuvré pendant près de deux décennies comme conseiller à I'Association canadienne de gestion des achats.

Gilles Paché est professeur de Sciences de Gestion à AixMarseille Université, où il occupe la fonction de directeur adjoint du Centre de Recherche sur le Transport et la Logistique (CRET-LOG), et de directeur du Service Commun des Presses Universitaires. Depuis le milieu des années 1980, il a publié ou édité une quinzaine d'ouvrages académiques, et il est l'auteur de plus de 350 articles, communications et chapitres d'ouvrage. Ses thématiques de recherche concernent principalement le management stratégique des entreprises industrielles et commerciales, les organisations en réseau, la gouvernance des clubs de football et le fonctionnement des chaînes logistiques multi-acteurs.

\section{Références}

Abbad, H. 2014. « La gestion mutualisée des approvisionnements : mythe ou réalités ? » Logistique \& Management 22 (2) : 41-50.

Andreff, W. 2007. "Régulation et institutions en économie du sport. » Revue de la Régulation (1) : 1-19.

Becker, G. 1964. Human Capital: A Theoretical and Empirical Analysis, with Special Reference to Education. Chicago, IL: University of Chicago Press.

Bourg, J.-F., et J.-J. Gouguet. 2012. Économie du sport. Paris : La Découverte, 3e éd.

Brach, B. 2012. "Who is Lionel Messi? A Comparative Study of Diego Maradona and Lionel Messi." International Journal of Cultural Studies 15 (4): 415-428.

Brandenburger, A., and B. Nalebuff. 1996. Co-Opetition: A Revolution Mindset that Combines Competition and Cooperation. New York: HarperCollins.

Camman, C., M. Monnet, G. Guieu, et L. Livolsi. 2013. « Les stratégies d'acteurs dans la mutualisation logistique, Logistique \& Management. » 21 (3): 57-75.

Christaller, W. 1933/2009. Die zentralen Orte in Süddeutschland. Darmstadt: WBG Verlag. 
Cox, A. 2001. "The Power Perspective in Procurement and Supply Management." Journal of Supply Chain Management 37 (1): 4-7.

Demazière, D., et M. Jouvenet. 2011. «Les agents sportifs dans le football : rôles professionnels et régulations de l'activité. » Dans Football, Europe et régulations, éd. par G. Robin, 191199. Lille : Presses Universitaires du Septentrion.

Drut, B. 2014. Économie du football professionnel, 2e éd. Paris : La Découverte.

Faure, J.-M., et C. Suaud. 1999. Le football professionnel à la française. Paris : Presses Universitaires de France.

des Garets, V., P. Nicholson, et G. Paché. 2016. " Jouer collectif pour faire prospérer une industrie : le football professionnel au défi du modèle de la ligue fermée. » Dans Génération marketing et sciences de gestion, éd. par C. Fournier et V. des Garets, 340-355. Paris : Economica.

Ghaderi, H., S. Darestani, Z. Leman, and M. Ismail. 2012. "Horizontal Collaboration in Logistics: A Feasible Task for Group Purchasing." International Journal of Procurement Management 5 (1): 43-54.

Gouguet, J.-J., et D. Primault. 2002. « Analyse économique du fonctionnement du marché des transferts dans le football professionnel. » Revue des Affaires Européennes (3): 305-323.

Graves, J. 1998. " Controlling Athletes with the Draft and the Salary Cap: Are Both Necessary?" Sports Lawyers Journal 5 (1): 185-203.

Helleu, B., et C. Durand. 2007. « La métropolisation du sport professionnel en Europe et en Amérique du Nord : une approche comparative. » Mappemonde (88) : 1-15.

Hoehn, T., and S. Szymanski. 1999. "The Americanization of European Football." Economic Policy 14 (28) : 205-240.

Keefer, Q. 2016. "The 2011 NFL Collective Bargaining Agreement and Drafted Player Compensation." International Journal of Sport Finance 11 (3): 221-231.

Kraljic, P. 1983. "Purchasing Must become Supply Management. " Harvard Business Review 61 (5): 109-117.

Le Roy, F., et S. Yami, éds. 2009. Management stratégique de la concurrence. Paris : Dunod.

Llorca, M., et T. Teste. 2016. «Dépenses salariales et performance dans l'industrie du football. " Revue Française d'Économie 31 (2): 125-145.

Lonsdale, C. 2004. "Player Power: Capturing Value in the English Football Supply Network." Supply Chain Management: An International Journal 9 (5): 383-391.

Maloni, M., and W. Benton. 2000. "Power Influences in the Supply Chain." Journal of Business Logistics 21 (1): 49-73.

Marquès, P. 2011. Stratégies relationnelles et performances : le cas du football professionnel européen, Thèse de doctorat en Sciences de Gestion, Université Montpellier I, février.

Marquès, P. 2015. « Déterminants et modalités du répertoire concurrentiel : une étude empirique du football professionnel européen. » Recherches en Sciences de Gestion (109): 25-45.

Marquès, P., et de J. Bontin. 2014. «La guerre des étoiles : le business des stars du ballon rond. "Dans Coopétition : s'allier à ses concurrents pour gagner, éd. J. Granata et P. Marquès, 155-189. Paris : Pearson.
Meyssonnier, F., et M. Mincheneau. 2013. «Le contrôle de gestion des clubs de football professionnel, Finance Contrôle Stratégie. » 16 (4) : 1-20.

Montalan, M.-A., et B. Vincent. 2011. "Prise en compte de la complexité dans la mutualisation des achats hospitaliers. "Projectics Proyéctica Projectique (8-9) : 107-116.

Montoya-Torres, J., A. Muñoz-Villamizar, and C. Vega-Mejía. 2016. "On the Impact of Collaborative Strategies for Goods Delivery in City Logistics." Production Planning \& Control 27 (6): 443-455.

Neale, W. 1964. "The Peculiar Economics of Professional Sports: A Contribution to the Theory of the Firm in Sporting Competition and in Market Competition." Quarterly Journal of Economics 78 (1): 1-14.

Paché, G., et G. N'Goala. 2011. « Les stratégies de création et d'appropriation de la valeur dans un contexte d'incertitude majeure : le cas du football professionnel. » Management \& Avenir (46) : 53-78.

Schotté, M. 2016. " 'Acheter' et 'vendre' un joueur : I'institution du transfert dans le football professionnel. » Marché \& Organisations (27) : 149-165.

Senkel, M.-P., B. Durand, et T. Hoa Vo. 2013. « La mutualisation logistique : entre théories et pratiques. » Logistique \& Management 21 (1) : 19-30.

Sloane, P. 1969. "The Labour Market in Professional Football." British Journal of Industrial Relations 7 (2) : 181-199.

Sloane, P. 2015. "The Economics of Professional Football Revisited." Scottish Journal of Political Economy 62 (1): 1-7.

Taylor, M. 2016. "Les joueurs de football sont-ils des esclaves ? Conditions d'emploi dans le milieu du football professionnel en Angleterre et au Pays de Galles (19451961). » Le Mouvement Social (254) : 47-68.

Toussaint, J.-P. 2015. Football. Paris : Les Éditions de Minuit.

van Weele, A. 2014. Purchasing and Supply Chain Management, $6^{\text {th }}$ ed. Boston, MA: Cengage Learning.

Vrooman, J. 1995. "A General Theory of Professional Sports Leagues." Southern Economic Journal 61 (4): 971-990.

Wahl, A., et P. Lanfranchi. 1995. Les footballeurs professionnels des années trente à nos jours. Paris: Hachette.

Zoudji, B., et D. Rey, éds. 2015. Le football dans tous ses états : Évolutions et questions d'actualité. Bruxelles : De Boeck.

\section{Sitographie}

http://www.aslog.org/ (consulté le 29 décembre 2015)

http://www.chargeurspointedebretagne.com/ (consulté le 11 mars 2016)

http://www.forbes.com/ (consulté le 27 janvier 2016)

http://www.juritravail.com/ (consulté le 16 avril 2016)

http://www.lemonde.fr/ (consulté le 12 novembre 2015)

http://sport24.lefigaro.fr/ (consulté 14 mai 2016)

http://www.transfermarkt.fr/ (consulté le 31 mai 2016) 